\title{
A Method to Polarize Stored Antiprotons to a High Degree
}

\author{
F. Rathmann,,${ }^{1, *}$ P. Lenisa, ${ }^{2}$ E. Steffens,${ }^{3}$ M. Contalbrigo, ${ }^{2}$ P. F. Dalpiaz, ${ }^{2}$ A. Kacharava, ${ }^{3}$ A. Lehrach, ${ }^{1}$ B. Lorentz, ${ }^{1}$ \\ R. Maier, ${ }^{1}$ D. Prasuhn, ${ }^{1}$ and H. Ströher ${ }^{1}$ \\ ${ }^{1}$ Institut für Kernphysik, Forschungzentrum Jülich, 52428 Jülich, Germany \\ ${ }^{2}$ Università di Ferrara and INFN, 44100 Ferrara, Italy \\ ${ }^{3}$ Physikalisches Institut II, Universität Erlangen-Nürnberg, 91058 Erlangen, Germany
}

(Received 9 October 2004; published 6 January 2005)

\begin{abstract}
Polarized antiprotons can be produced in a storage ring by spin-dependent interaction in a purely electron-polarized hydrogen gas target. The polarizing process is based on spin transfer from the polarized electrons of the target atoms to the orbiting antiprotons. After spin filtering for about two beam lifetimes at energies $T \approx 40-170 \mathrm{MeV}$ using a dedicated large acceptance ring, the antiproton beam polarization would reach $P=0.2-0.4$. Polarized antiprotons would open new and unique research opportunities for spin-physics experiments in $\bar{p} p$ interactions.
\end{abstract}

DOI: 10.1103/PhysRevLett.94.014801

PACS numbers: 29.27.Hj, 24.70.+s, 29.25.Pj

For more than two decades, physicists have tried to produce beams of polarized antiprotons [1]. Conventional methods like atomic beam sources (ABS), appropriate for the production of polarized protons and heavy ions, cannot be applied, since antiprotons annihilate with matter. Polarized antiprotons have been produced from the decay in flight of $\bar{\Lambda}$ hyperons at Fermilab. The achieved intensities with antiproton polarizations $P>0.35$ never exceeded $1.5 \times 10^{5} \mathrm{~s}^{-1}$ [2]. Scattering of antiprotons off a liquid hydrogen target could yield polarizations of $P \approx$ 0.2 , with beam intensities of up to $2 \times 10^{3} \mathrm{~s}^{-1}$ [3]. Unfortunately, both approaches do not allow efficient accumulation in a storage ring, which would greatly enhance the luminosity. Spin splitting using the Stern-Gerlach separation of the given magnetic substates in a stored antiproton beam was proposed in 1985 [4]. Although the theoretical understanding has much improved since then [5], spin splitting using a stored beam has yet to be observed experimentally.

Interest in the polarization of antiprotons has recently been stimulated by a proposal to build a High Energy Storage Ring (HESR) for antiprotons at the new Facility for Antiproton and Ion Research (FAIR) at the Gesellschaft für Schwerionenforschung (GSI) in Darmstadt [6]. A Letter of intent for spin-physics experiments has been submitted by the PAX Collaboration [7] to employ a polarized antiproton beam incident on a polarized internal storage cell target [8]. A beam of polarized antiprotons would enable new experiments, such as the first direct measurement of the transversity distribution of the valence quarks in the proton, a test of the predicted opposite sign of the Sivers-function-related to the quark distribution inside a transversely polarized nucleon-in Drell-Yan as compared to semi-inclusive deep-inelastic scattering, and a first measurement of the moduli and the relative phase of the timelike electric and magnetic form factors $G_{\mathrm{E}, \mathrm{M}}$ of the proton [7].
In 1992 an experiment at the Test Storage Ring (TSR) at MPI Heidelberg showed that an initially unpolarized stored $23 \mathrm{MeV}$ proton beam can be polarized by spin-dependent interaction with a polarized hydrogen gas target [9-11]. In the presence of polarized protons of magnetic quantum number $m=\frac{1}{2}$ in the target, beam protons with $m=\frac{1}{2}$ are scattered less often than those with $m=-\frac{1}{2}$, which eventually caused the stored beam to acquire a polarization parallel to the proton spin of the hydrogen atoms during spin filtering. In an analysis by Meyer three different mechanisms were identified that add up to the measured result [12]. One of these mechanisms is spin transfer from the polarized electrons of the hydrogen gas target to the circulating protons. Horowitz and Meyer derived the spintransfer cross section $p+\vec{e} \rightarrow \vec{p}+e$ (using $c=\hbar=1$ ) [13],

$$
\sigma_{e_{\|}}=-\frac{4 \pi \alpha^{2}(1+a) m_{e}}{p^{2} m_{p}} \cdot C_{0}^{2} \cdot \frac{v}{2 \alpha} \cdot \sin \left[\frac{2 \alpha}{v} \ln \left(2 p a_{0}\right)\right],
$$

where $\alpha$ is the fine-structure constant, $a$ is the anomalous magnetic moment of the proton, $m_{e}$ and $m_{p}$ are the rest mass of electron and proton, $p$ is the momentum in the c.m. system, $a_{0}=52900 \mathrm{fm}$ is the Bohr radius, and $C_{0}^{2}=$ $2 \pi \eta /[\exp (2 \pi \eta)-1]$ is the square of the Coulomb wave function at the origin. The Coulomb parameter $\eta$ is given by $\eta=-z \alpha / v$ (for antiprotons, $\eta$ is positive). $z$ is the beam charge number and $v$ the relative velocity of particle and projectile.

In the following we evaluate a concept for a dedicated antiproton polarizer ring (AP). Antiprotons would be polarized by the spin-dependent interaction in an electronpolarized hydrogen gas target. This spin-transfer process is calculable, whereas due to the absence of polarized antiproton beams in the past, a measurement of the spindependent $\bar{p} p$ interaction is still lacking, and only theoretical models exist [14]. The polarized antiprotons would 
be subsequently transferred to an experimental storage ring (ESR) for measurements (Fig. 1). Both the AP and the ESR should be operated as synchrotrons with electron cooling to counteract emittance growth. In both rings the beam polarization should be preserved during acceleration without loss [15]. The longitudinal spin-transfer cross section is twice as large as the transverse one [12], $\sigma_{e_{\|}}=2 \cdot \sigma_{e_{\perp}}$; the stable spin direction of the beam at the location of the polarizing target should therefore be longitudinal as well, which requires a Siberian snake in a straight section opposite the polarizing target [18].

A hydrogen gas target of suitable substate population represents a dense target of quasifree electrons of high polarization and areal density. Such a target can be produced by injection of two hyperfine states with magnetic quantum numbers $\left|m_{J}=+\frac{1}{2}, m_{I}=+\frac{1}{2}\right\rangle$ and $\left|+\frac{1}{2},-\frac{1}{2}\right\rangle$ into a strong longitudinal magnetic holding field of about $B_{\|}=300 \mathrm{mT}$. The maximum electron and nuclear target polarizations in such a field are $Q_{e}=0.993$ and $Q_{z}=$ 0.007 [19]. Polarized atomic beam sources presently produce a flux of hydrogen atoms of about $q=1.2 \times$ $10^{17}$ atoms/s in two hyperfine states [20]. Our model calculation for the polarization buildup assumes a moderate improvement of $20 \%$, i.e., a flow of $q=1.5 \times$ $10^{17}$ atoms/s.

The beam lifetime in the AP can be expressed as function of the Coulomb-loss cross section $\Delta \sigma_{C}$ and the total hadronic $\bar{p} p$ cross section $\sigma_{\text {tot }}$,

$$
\tau_{\mathrm{AP}}=\frac{1}{\left(\Delta \sigma_{C}+\sigma_{\mathrm{tot}}\right) \cdot d_{t} \cdot f_{\mathrm{AP}}} .
$$

The density $d_{t}$ of a storage cell target depends on the flow of atoms $q$ into the feeding tube of the cell, its length along the beam $L_{\text {beam }}$, and the total conductance $C_{\text {tot }}$ of the storage cell $d_{t}=\frac{1}{2} \frac{L_{\mathrm{beam}} \cdot q}{C_{\mathrm{tot}}}$ [8]. The conductance of a cylindrical tube $C_{\circ}$ for a gas of mass $M$ in the regime of molecular flow (mean free path large compared to the dimensions of the tube) as a function of its length $L$, diameter $d$, and temperature $T$, is given by $C_{\circ}=3.8 \cdot \sqrt{\frac{T}{M}}$.

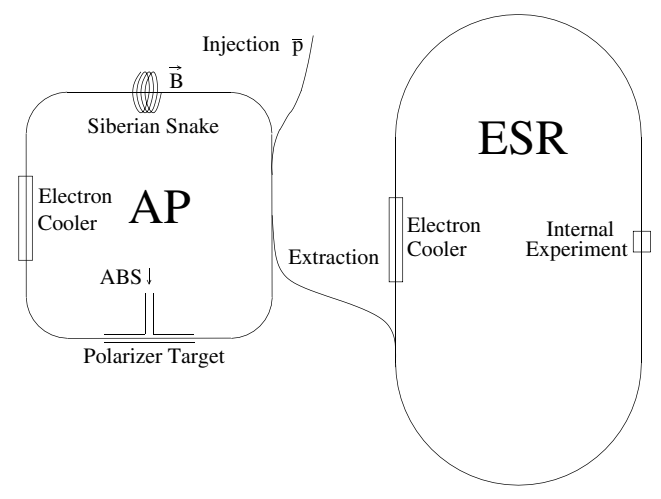

FIG. 1. Antiproton polarizer (AP) and experimental storage ring (ESR).
$\frac{d^{3}}{L+\frac{4}{3} \cdot d}$. The total conductance $C_{\mathrm{tot}}$ of the storage cell is given by $C_{\mathrm{tot}}=C_{\circ}^{\text {feed }}+2 \cdot C_{\circ}^{\text {beam }}$, where $C_{\circ}^{\text {feed }}$ denotes the conductance of the feeding tube and $C_{\circ}^{\text {beam }}$ the conductance of one-half of the beam tube. The diameter of the beam tube of the storage cell should match the ring acceptance angle $\Psi_{\text {acc }}$ at the target, $d_{\text {beam }}=2 \cdot \Psi_{\text {acc }} \cdot \beta$, where for the $\beta$ function at the target, we use $\beta=\frac{1}{2} L_{\text {beam }}$. One can express the target density in terms of the ring acceptance, $d_{t} \equiv$ $d_{t}\left(\Psi_{\text {acc }}\right)$, where the other parameters used in the calculation are listed in Table I.

The Coulomb-loss cross section $\Delta \sigma_{C}$ (using $c=\hbar=1$ ) can be derived analytically in terms of the square of the total energy $s$ by integration of the Rutherford cross section, taking into account that only those particles are lost that undergo scattering at angles larger than $\Psi_{\text {acc }}$,

$$
\Delta \sigma_{C}\left(\Psi_{\text {acc }}\right)=4 \pi \alpha^{2} \frac{\left(s-2 m_{\bar{p}}^{2}\right)^{2} 4 m_{\bar{p}}^{2}}{s^{2}\left(s-4 m_{\bar{p}}^{2}\right)^{2}}\left(\frac{1}{\Psi_{\text {acc }}^{2}}-\frac{s}{4 m_{\bar{p}}^{2}}\right) .
$$

The total hadronic cross section is parametrized using a function inversely proportional to the Lorentz parameter $\beta_{\text {lab. }}$. Based on the $\bar{p} p$ data [21] the parametrization $\sigma_{\text {tot }}=$ $\frac{75.5}{\beta_{\text {lab }}}(\mathrm{mb})$ yields a description of $\sigma_{\text {tot }}$ with $\approx 15 \%$ accuracy up to $T \approx 1000 \mathrm{MeV}$. The AP revolution frequency is given by $f_{\mathrm{AP}}=\frac{\beta_{\mathrm{lab}} \cdot c}{L_{\mathrm{AP}}}$. The resulting beam lifetime in the AP as function of the kinetic energy $T$ is depicted in Fig. 2 for different acceptance angles $\Psi_{\text {acc }}$.

The buildup of polarization due to the spin-dependent $\bar{p} e$ interaction in the target [Eq. (1)] as function of time $t$ is described by

$$
P(t)=\tanh \left(\frac{t}{\tau_{p}}\right), \quad \text { where } \tau_{p}=\frac{1}{\sigma_{e_{\|}} d_{t} f_{\mathrm{AP}} Q_{e}}
$$

denotes the polarization buildup time. The time dependence of the beam intensity is described by

$$
I(t)=I_{0} \cdot \exp \left(-\frac{t}{\tau_{\mathrm{AP}}}\right) \cdot \cosh \left(\frac{t}{\tau_{p}}\right),
$$

where $I_{0}=N_{\bar{p}}^{\mathrm{AP}} \cdot f_{\mathrm{AP}}$.

TABLE I. Parameters of the AP and the polarizing target section.

\begin{tabular}{lcc}
\hline \hline Circumference of AP & $L_{\mathrm{AP}}$ & $150 \mathrm{~m}$ \\
$\beta$ function at target & $\beta$ & $0.2 \mathrm{~m}$ \\
Radius of vacuum chamber & $r$ & $5 \mathrm{~cm}$ \\
Gap height of magnets & $2 g$ & $14 \mathrm{~cm}$ \\
\hline ABS flow into feeding tube & $q$ & $1.5 \times 10^{17}$ atoms $/ \mathrm{s}$ \\
Storage cell length & $L_{\text {beam }}$ & $40 \mathrm{~cm}$ \\
Feeding tube diameter & $d_{\text {feed }}$ & $1 \mathrm{~cm}$ \\
Feeding tube length & $L_{\text {feed }}$ & $15 \mathrm{~cm}$ \\
Longitudinal holding field & $B_{\|}$ & $300 \mathrm{mT}$ \\
Electron polarization & $Q_{e}$ & 0.9 \\
Cell temperature & $T$ & $100 \mathrm{~K}$ \\
\hline \hline
\end{tabular}




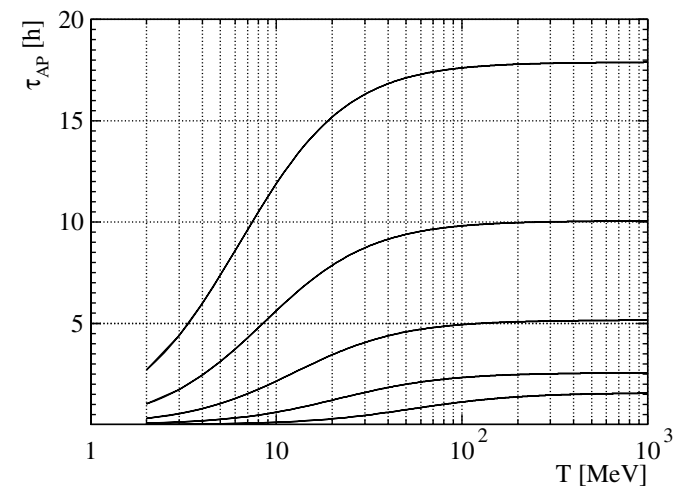

FIG. 2. Beam lifetime in the AP as function of kinetic energy $T$. From top to bottom the lines denote $\Psi_{\text {acc }}=50,40,30,20$, and $10 \mathrm{mrad}$.

The quality of the polarized antiproton beam can be expressed in terms of the figure of merit (FOM) [22]

$$
\operatorname{FOM}(t)=P(t)^{2} \cdot I(t)
$$

The optimum interaction time $t_{\mathrm{opt}}$, where $\operatorname{FOM}(t)$ reaches the maximum, is given by $\frac{d}{d t} \operatorname{FOM}(t)=0$. For the situation discussed here, $t_{\mathrm{opt}}=2 \cdot \tau_{\mathrm{AP}}$ constitutes a good approximation that deviates from the true values by at most $3 \%$. The magnitude of the antiproton beam polarization $P\left(t_{\mathrm{opt}}\right)$ based on electron spin transfer [Eq. (4)] is depicted in Fig. 3 as function of beam energy $T$ for different acceptance angles $\Psi_{\text {acc }}$.

The number of antiprotons stored in the AP may be limited by space-charge effects. With an antiproton production rate of $R=10^{7} \bar{p} / \mathrm{s}$, the number of antiprotons available at the beginning of the filtering procedure corresponds to

$$
N_{\bar{p}}^{\mathrm{AP}}(t=0)=R \cdot 2 \cdot \tau_{\mathrm{AP}}
$$

The individual particle limit in the AP is given by [23]

$$
N_{\text {ind. }}=2 \pi \varepsilon \beta_{\text {lab }}^{2} \gamma_{\text {lab }}^{3}\left(r_{p} F\right)^{-1} \Delta Q,
$$

where $\varepsilon=\Psi_{\text {acc }}^{2} \cdot \beta$ denotes the vertical and horizontal

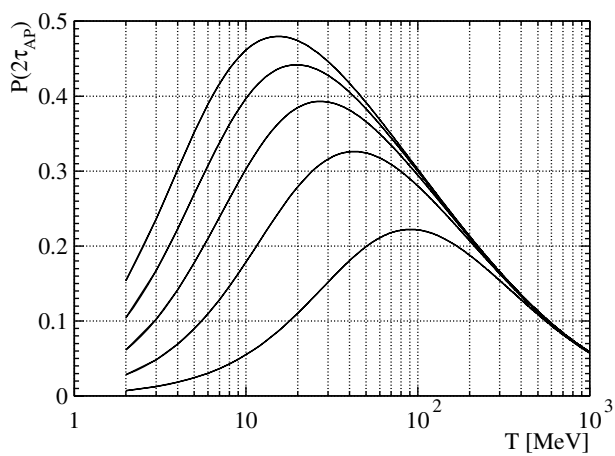

FIG. 3. Antiproton beam polarization $P\left(2 \cdot \tau_{\mathrm{AP}}\right)$ [Eq. (4)] as function of beam energy for different acceptance angles $\Psi_{\text {acc }}$. (Lines are organized as in Fig. 2.) beam emittance, $\beta_{\text {lab }}$ and $\gamma_{\text {lab }}$ are the Lorentz parameters, $r_{p}=1.5347 \times 10^{-18} \mathrm{~m}$ is the classical proton radius, and $\Delta Q=0.01$ is the allowed incoherent tune spread. The form factor $F$ for a circular vacuum chamber [23] is given by $F=1+\left(a_{y} \cdot \frac{a_{x}+a_{y}}{r^{2}}\right) \cdot \varepsilon_{2} \cdot\left(\gamma_{\text {lab }}^{2}-1\right) \cdot \frac{r^{2}}{g^{2}}$, where the mean semiminor horizontal $(x)$ and vertical $(y)$ beam axes $a_{x, y}=\sqrt{\varepsilon \cdot \beta_{x, y}}$ are calculated from the mean horizontal and vertical $\beta$ functions $\beta_{x, y}=L_{\mathrm{AP}} \cdot(2 \pi \nu)^{-1}$ for a betatron-tune $\nu=3.6$. For a circular vacuum chamber and straight magnet pole pieces, the image force coefficient $\varepsilon_{2}=0.411$. The parameter $r$ denotes the radius of the vacuum chamber and $g$ half of the height of the magnet gaps (Table I).

The optimum beam energies for different acceptance angles at which the polarization buildup works best, however, cannot be obtained from the maxima in Fig. 3. In order to find these energies, one has to evaluate at which beam energies the FOM [Eq. (6)], depicted in Fig. 4, reaches a maximum. The optimum beam energies for polarization buildup in the AP are listed in Table II. The limitations due to space charge, $N_{\bar{p}}^{\mathrm{AP}}>N_{\text {ind. }}$ [Eqs. (7) and (8)], are visible as kinks in Fig. 4 for the acceptance angles $\Psi_{\text {acc }}=40$ and $50 \mathrm{mrad}$; however, the optimum energies are not affected by space charge.

Spin filtering in a pure electron target greatly reduces the beam losses because $\sigma_{\text {tot }}$ disappears and Coulomb scattering angles in $\bar{p} e$ collisions do not exceed $\Psi_{\text {acc }}$ of any storage ring. With stationary electrons stored in a Penning trap, densities of about $10^{12}$ electrons $/ \mathrm{cm}^{2}$ may be reached in the future [24]. A typical electron cooler operated at $10 \mathrm{kV}$ with polarized electrons of intensity $\approx$ $1 \mathrm{~mA} \quad\left(I_{e} \approx 6.2 \times 10^{15}\right.$ electrons $\left./ \mathrm{s}\right) \quad[25], \quad A=1 \mathrm{~cm}^{2}$ cross section, and $l=5 \mathrm{~m}$ length reaches $d_{t}=$ $I_{e} \cdot l \cdot\left(\beta_{\text {lab }} \mathrm{cA}\right)^{-1}=5.2 \times 10^{8}$ electrons $/ \mathrm{cm}^{2}$, which is 6 orders of magnitude short of the electron densities achievable with a neutral hydrogen gas target. For a pure electron target, the spin-transfer cross section is $\sigma_{e_{\|}}=$ $670 \mathrm{mb}$ (at $T=6.2 \mathrm{MeV}$ ) [13], about a factor 15 larger

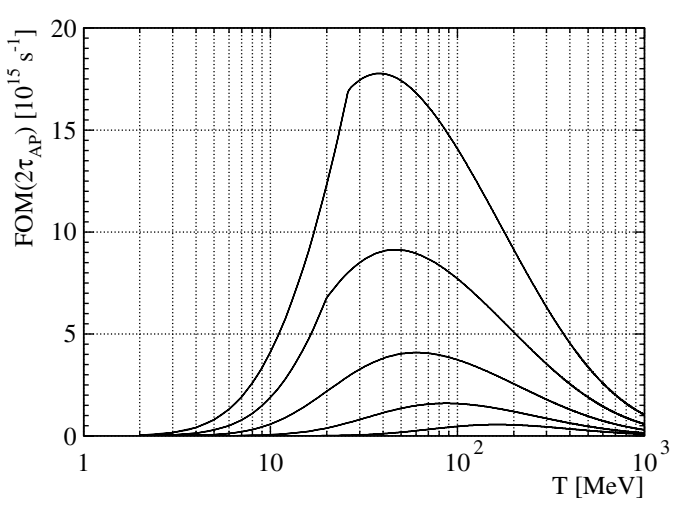

FIG. 4. Figure of merit for the polarized antiproton beam for filtering times $t=2 \cdot \tau_{\mathrm{AP}}$ as function of beam energy. The parameters associated with the maxima are summarized in Table II. (Lines are organized as in Fig. 2.) 
TABLE II. Kinetic beam energies where the polarized antiproton beam in the AP reaches the maximum FOM for different acceptance angles.

\begin{tabular}{lccc}
\hline \hline$\Psi_{\mathrm{acc}}(\mathrm{mrad})$ & $T(\mathrm{MeV})$ & $\tau_{\mathrm{AP}}(\mathrm{h})$ & $P\left(2 \tau_{\mathrm{AP}}\right)$ \\
\hline 10 & 167 & 1.2 & 0.19 \\
20 & 88 & 2.2 & 0.29 \\
30 & 61 & 4.6 & 0.35 \\
40 & 47 & 9.2 & 0.39 \\
50 & 39 & 16.7 & 0.42 \\
\hline \hline
\end{tabular}

than the cross sections associated with the optimum energies using a gas target (Table II). One can therefore conclude that with present day technologies, both above discussed alternatives are no match for spin filtering using a polarized gas target.

In order to estimate the luminosities available for the ESR, we use the parameters of the $\operatorname{HESR}\left(L_{\mathrm{HESR}}=\right.$ $440 \mathrm{~m})$. After spin filtering in the AP for $t_{\mathrm{opt}}=2 \cdot \tau_{\mathrm{AP}}$, the number of polarized antiprotons transferred to HESR is $N_{\bar{p}}^{\mathrm{AP}}(t=0) / e^{2}$ [Eq. (7)]. The beam lifetime in the HESR at $T=15 \mathrm{GeV}$ for an internal polarized hydrogen gas target of $d_{t}=7 \times 10^{14} \mathrm{~cm}^{-2}$ is about $\tau_{\mathrm{HESR}}=12 \mathrm{~h}$ [Eq. (2)], where the target parameters from Table I were used, a cell diameter $d_{\text {beam }}=0.8 \mathrm{~cm}$, and $\sigma_{\text {tot }}=50 \mathrm{mb}$. Subsequent transfers from the AP to the HESR can be employed to accumulate antiprotons. Eventually, since $\tau_{\text {HESR }}$ is finite, the average number of antiprotons reaches equilibrium, $\bar{N}_{\bar{p}}^{\mathrm{HESR}}=R / e^{2} \cdot \tau_{\mathrm{HESR}}=5.6 \times 10^{10}$, independent of $\tau_{\mathrm{AP}}$. An average luminosity of $\overline{\mathcal{L}}=R /\left(e^{2} \cdot \sigma_{\mathrm{tot}}\right)=$ $2.7 \times 10^{31} \mathrm{~cm}^{-2} \mathrm{~s}^{-1}$ can be achieved, with antiproton beam polarizations depending on the AP acceptance angle $\Psi_{\text {acc }}$ (Table II).

We have shown that with a dedicated large acceptance antiproton polarizer ring ( $\Psi_{\text {acc }}=10$ to $50 \mathrm{mrad}$ ), beam polarizations of $P=0.2$ to 0.4 could be reached. The energies at which the polarization buildup works best range from $T=40$ to $170 \mathrm{MeV}$. In equilibrium, the average luminosity for double-polarization experiments in an experimental storage ring (e.g., HESR) after subsequent transfers from the AP could reach $\overline{\mathcal{L}}=2.7 \times$ $10^{31} \mathrm{~cm}^{-2} \mathrm{~s}^{-1}$.

The antiproton polarizer discussed here would provide highly polarized antiproton beams of unprecedented quality. In particular, the implementation of this option at the Facility for Antiproton and Ion Research would open new and unique research opportunities for spin-physics experiments in $\bar{p} p$ interactions at the HESR.

We would like to thank J. Haidenbauer and N.N. Nikolaev for many insightful discussions on the subject.

*Electronic address: f.rathmann@fz-juelich.de
[1] Proceedings of the Workshop on Polarized Antiprotons, Bodega Bay, CA, 1985, edited by A. D. Krisch, A. M. T. Lin, and O. Chamberlain, AIP Conf. Proc. No. 145 (AIP, New York, 1986).

[2] D. P. Grosnick et al., Nucl. Instrum. Methods Phys. Res., Sect. A 290, 269 (1990).

[3] H. Spinka et al., Proceedings of the 8th International Symposium on Polarization Phenomena in Nuclear Physics, Bloomington, Indiana, 1994, edited by E.J. Stephenson and S.E. Vigdor, AIP Conf. Proc. No. 339 (AIP, New York, 1995), p. 713.

[4] T. O. Niinikoski and R. Rossmanith, Nucl. Instrum. Methods Phys. Res., Sect. A 255, 460 (1987).

[5] P. Cameron et al., Proceedings of the 15th International Spin Physics Symposium, Upton, New York, 2002, edited by Y. I. Makdisi, A. U. Luccio, and W.W. MacKay, AIP Conf. Proc. No. 675 (AIP, New York, 2003), p. 781.

[6] Conceptual Design Report for An International Facility for Antiproton and Ion Research. Available from http:// www.gsi.de/GSI-Future/cdr.

[7] P. Lenisa and F. Rathmann (spokespersons), "AntiprotonProton Scattering Experiments with Polarization," Letter of intent for the HESR at FAIR, Jülich, 2004, and references therein. Available from http://www.fz-juelich.de/ ikp/pax.

[8] E. Steffens and W. Haeberli, Rep. Prog. Phys. 66, 1887 (2003).

[9] F. Rathmann et al., Phys. Rev. Lett. 71, 1379 (1993).

[10] K. Zapfe et al., Rev. Sci. Instrum. 66, 28 (1995).

[11] K. Zapfe et al., Nucl. Instrum. Methods Phys. Res., Sect. A 368, 627 (1996).

[12] H. O. Meyer, Phys. Rev. E 50, 1485 (1994).

[13] C. J. Horowitz and H. O. Meyer, Phys. Rev. Lett. 72, 3981 (1994).

[14] V. Mull and K. Holinde, Phys. Rev. C 51, 2360 (1995).

[15] Preservation of beam polarization is routinely accomplished at COSY-Jülich [16], and at higher energies at the AGS of BNL [17].

[16] A. Lehrach et al., see p. 153 of Ref. [5].

[17] F.Z. Khiari et al., Phys. Rev. D 39, 45 (1989); H. Huang et al., Phys. Rev. Lett. 73, 2982 (1994); M. Bai et al., Phys. Rev. Lett. 80, 4673 (1998).

[18] Ya. S. Derbenev et al., Part. Accel. 8, 115 (1978); A. Lehrach and R. Maier, Proceedings of the 2001 Particle Accelerator Conference, Chicago, Illinois, 2001, edited by P. Lucas and S. Webber (IEEE, Piscataway, NJ, 2001), p. 2566.

[19] W. Haeberli, Annu. Rev. Nucl. Sci. 17, 373 (1967).

[20] A. Zelenski et al., Nucl. Instrum. Methods Phys. Res., Sect. A (to be published).

[21] S. Eidelman et al., Phys. Lett. B 592, 1 (2004).

[22] G. G. Ohlsen and P. W. Keaton, Nucl. Instrum. Methods 109, 41 (1973).

[23] C. Bovet, R. Gouiran, I. Gumowski, and K. H. Reich, CERN Report No. CERN/MPS-SI/Int. DL/70/4, 1970.

[24] J. H. Malmberg et al., Non-Neutral Plasma Physics, edited by C.W. Roberson and C.F. Driscoll (AIP, New York, 1988), p. 28.

[25] J. Grames et al., see p. 1047 of Ref. [5]. 